\title{
Hearing loss due to metastasis of gastric cancer to temporal bone: A case report
}

\author{
XIANGMING CAO $^{1,2^{*}}$, FANGBO CUI $^{3 *}$, JIA WEI $^{2}$, QING WANG $^{2}$, LI CHUN DENG $^{1}$, \\ BAO RUI LIU ${ }^{2}$ and WEI SHENG SHEN ${ }^{1}$
}

\begin{abstract}
${ }^{1}$ Department of Oncology, The Affiliated Jiangyin Hospital of Southeast University Medical College, Jiangyin, Jiangsu 214400; ${ }^{2}$ The Comprehensive Cancer Center of Nanjing Drum Tower Hospital, The Affiliated Hospital of Nanjing University Medical School and Clinical Cancer Institute of Nanjing University, Nanjing, Jiangsu 210008; ${ }^{3}$ Department of Oncology, The People's Hospital of Ma Anshan, Ma Anshan, Anhui 243000, P.R. China
\end{abstract}

Received November 18, 2014; Accepted September 29, 2015

DOI: $10.3892 / \mathrm{ol} .2016 .4084$

\begin{abstract}
Metastatic temporal bone tumors are rare, and tend to be asymptomatic. The clinical symptoms consist of aural discharge, bleeding, hearing loss and facial nerve paresis. The most common origin of the metastasis is breast cancer, and other sites of the primary tumor include the thyroid gland, brain, lungs, prostate and blood. Clinical reports of hearing loss due to gastric cancer metastatic to temporal bone are rare. In the present study, a case of gastric cancer metastasis to temporal bone without other organ involvement is described. The patient presented with the symptom of hearing loss, and the metastatic tumor was diagnosed by radiological imaging, including magnetic resonance imaging, computed tomography and bone scan.
\end{abstract}

\section{Introduction}

Despite a significant decline in its incidence and mortality in the past century (1), gastric cancer remains the fourth most common type of cancer, and the second leading cause of cancer-associated mortalities worldwide (2). In China, the majority of gastric cancer cases are diagnosed as metastatic or recurrent gastric cancer, which generally involves the liver,

Correspondence to: Professor Bao Rui Liu, The Comprehensive Cancer Center of Nanjing Drum Tower Hospital, The Affiliated Hospital of Nanjing University Medical School and Clinical Cancer Institute of Nanjing University, 321 Zhongshan Road, Nanjing, Jiangsu 210008, P.R. China

E-mail: Baoruiliu@nju.edu.cn

Mr. Wei Sheng Shen, Department of Oncology, The Affiliated Jiangyin Hospital of Southeast University Medical College, 163 Shoushan Road, Jiangyin, Jiangsu 214400, P.R. China

E-mail: shenweisheng2000@163.com

${ }^{*}$ Contributed equally

Key words: gastric cancer, temporal bone, metastasis, hearing loss lung, bone and abdominal cavity, and in 2008 the five-year survival rate was $<5 \%$ (3). The incidence of primary carcinoma in the temporal bone has been previously reported to be $1 / 2,000(4,5)$, while metastatic tumors in this organ are less common than primary tumors (2). In the present study, a rare case of hearing loss as a consequence of metastasis of gastric cancer to temporal bone without the involvement of other organs is reported.

\section{Case report}

In April 2014, a 55-year-old male was diagnosed with gastric cancer at The Affiliated Jiangyin Hospital of Southeast University Medical College (Jiangyin, China). The patient underwent radical total gastrectomy, and the histopathological diagnosis was gastric adenocarcinoma (Figs. 1 and 2). Immunohistochemistry demonstrated positive staining for cytokeratin (CK)7, CK8/18, CK20 and CKAE1/AE3, and negative staining for human epidermal growth factor receptor (Her)-2, P53 and vimentin. The patient was next subjected to postoperative chemotherapy, which consisted of six cycles of combination chemotherapy with oxaliplatin $\left(135 \mathrm{mg} / \mathrm{m}^{2}\right.$ on day 1 by intravenous (iv) infusion), epirubicin (EPI)-adriamycin (ADM) $\left(50 \mathrm{mg} / \mathrm{m}^{2}\right.$ on day 1) and 5-fluorouracil (FU) $\left(375-425 \mathrm{mg} / \mathrm{m}^{2}\right.$ on days $1-5$ via iv infusion). Concomitant palliative radiotherapy was administered to the gastric tumor bed and local lymphatic drainage area, with a total dose of $45 \mathrm{~Gy} / 25 \mathrm{~F}$. Bone scan (Symbia T16, Siemens AG, Munich, Germany) and abdominal computed tomography (CT) (Brilliance-64, Philips Healthcare, DA Best, The Netherlands) were performed as part of the regular follow-up. The patient experienced hearing loss 11 months later, and was admitted to the Department of Otolaryngology of The Affiliated Jiangyin Hospital of Southeast University Medical College for a full evaluation of the ear. Physical examination indicated normal external auditory canal and tympanic membrane. Bone conduction pure tone audiometry revealed mild $(35 \mathrm{~dB})$ loss of sensor neural hearing on the left side. Bone scan demonstrated uptake in the left temporal bone (Figs. 3 and 4). CT and magnetic resonance imaging (MRI) (Achieva 3.0T, Philips Healthcare) of the brain identified an enhancing lesion in the left petrous apex 


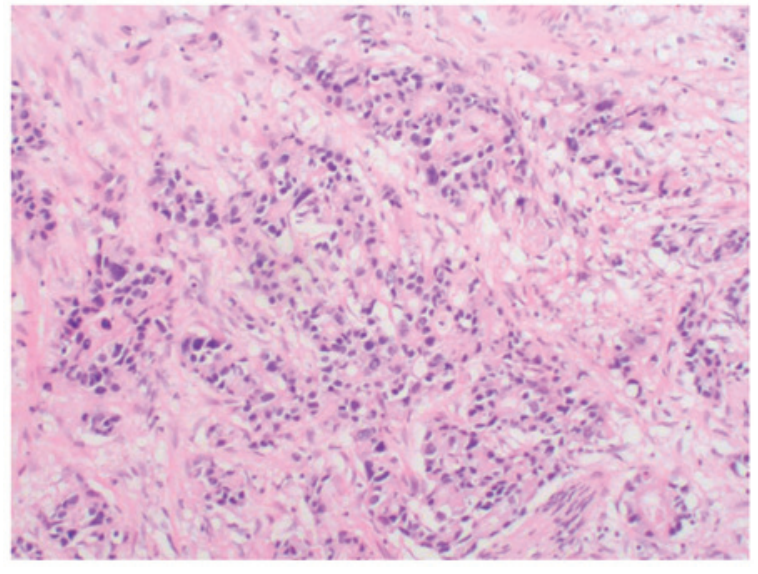

Figure 1. Hematoxylin and eosin-stained tissue section exhibiting gastric cancer with hyperchromatic nuclei and scant cytoplasm (magnification, x100).

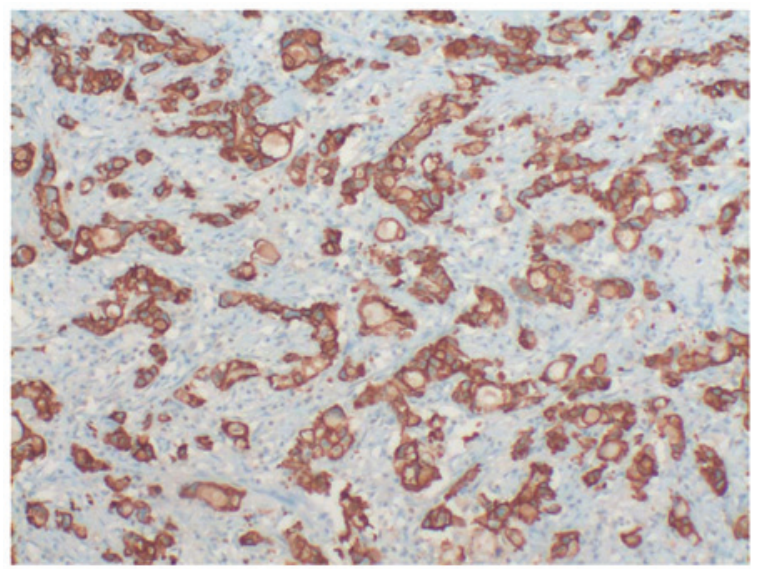

Figure 2. Immunohistochemistry indicated positive staining for cytokeratin 7.

(Figs. 5 and 6, respectively). Previous patients who were clinically diagnosed with metastasis to the temporal bone received radiotherapy with a total dose of $60 \mathrm{~Gy}$ for the treatment of the tumor mass (6). Therefore, this treatment was also applied to the patient of the present case report, who is currently under treatment and has been under observation for one year.

\section{Discussion}

Gastric cancer is one of the most common types of cancer worldwide (2). A significant number of patients with gastric cancer experience recurrence following radical gastrectomy (7). According to Baiocchi et al (8), the most frequent sites of recurrence were the local/regional lymph nodes (35.4\%), liver (24.3\%), peritoneum (30.3\%), lung (10.4\%) and intralumen (7.5\%). A small number of cases of metastasis to unusual sites such as skin, mouth and leptomeninges have been previously reported (9-11). Previous reports of hearing loss due to metastasis of gastric cancer are infrequent $(2,6)$. Ohno et al (12) and Kim et al (13) reported cases of metastasis of gastric cancer to meningeal carcinoma, which resulted in hearing loss. Joo et al (14) reported a case of gastric cancer manifesting as a solitary metastasis in the cerebellopontine angle that mimicked acoustic neuroma and caused hearing

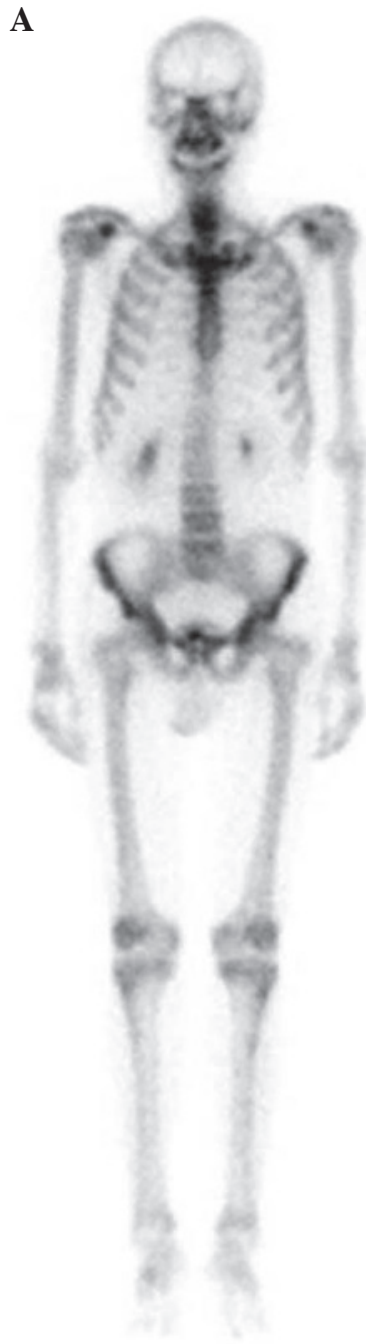

B

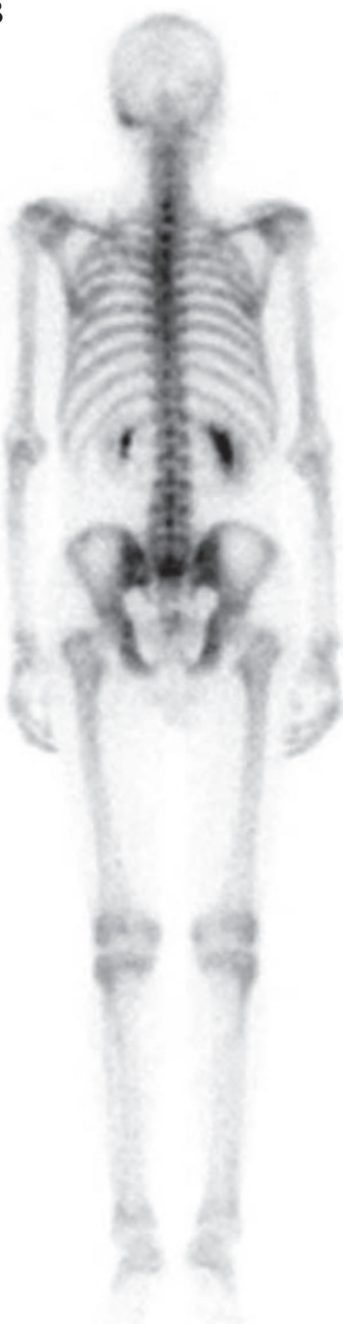

Figure 3. (A) Anterior and (B) posterior view of whole body bone scan, indicating uptake in the left temporal bone.

loss. In addition, a case of metastasis of gastric cancer to the internal auditory canal with multiple metastases to bone and liver has been previously reported (15). To the best of our knowledge, there are no reported cases of metastasis of Her-2 gastric cancer to temporal bone without the involvement of other organs that only present with the symptom of hearing loss.

Temporal bone cancer accounts for $<0.2 \%$ of all tumors involving the head and neck region (6). The majority of metastatic temporal bone tumors tend to be asymptomatic, and it may cause a variety of symptoms (6). Lionello et al (16) reported the most common clinical findings of temporal bone cancer, including aural discharge (24-100\%), bleeding (19-81\%), hearing loss (2-75\%) and facial nerve paresis (7-64\%).

The most common primary malignancy affecting the temporal bone is breast cancer. Other sites of primary tumors include the thyroid gland, brain, lungs, prostate and blood (17). The most common site of involvement in the temporal bone is the petrous apex, followed by the tegmen tympani, mastoid bone and internal auditory canal (17). Han et al (15) hypothesized that the circulation in the marrow spaces of the petrous apex facilitates the deposition of tumor 
A

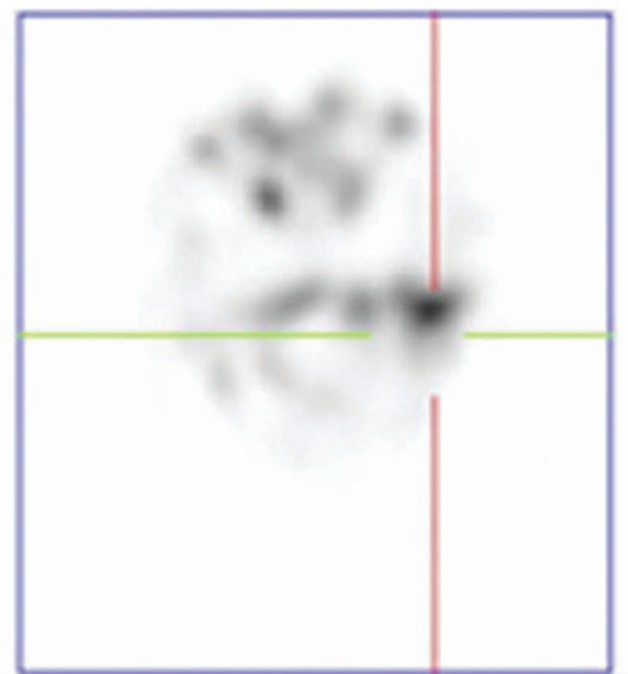

B

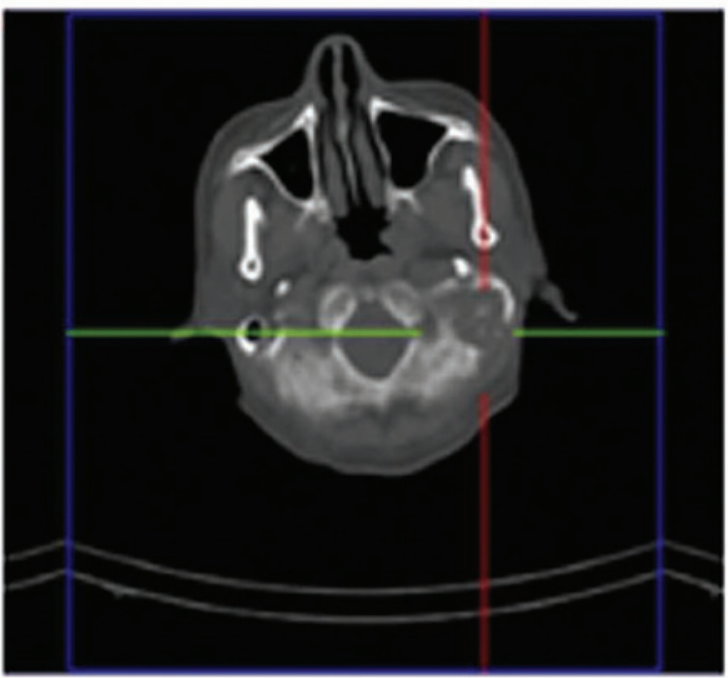

C

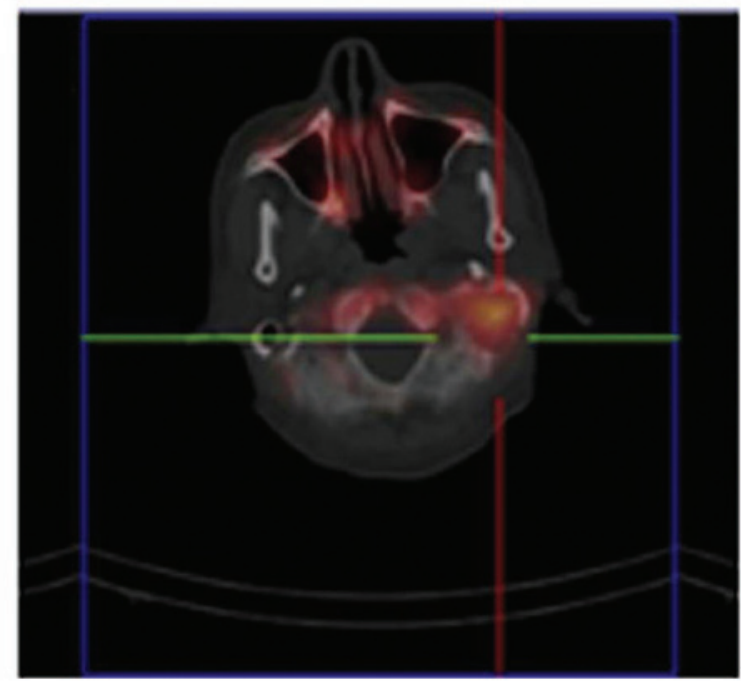

Figure 4. Bone scan of the skull revealed uptake in the left temporal bone. (A) ECT with corrected attenuation. (B) CT. (C) Fused CT and ECT images. CT, computed tomography; ECT, enhanced CT.

cells, which is unusual in pneumatized areas inflated by the tumor.

There are five possible patterns of tumor spreading from the primary site to the temporal bone $(5,6,15,17)$ : i) Hematogenous spreading; ii) directly spreading though the

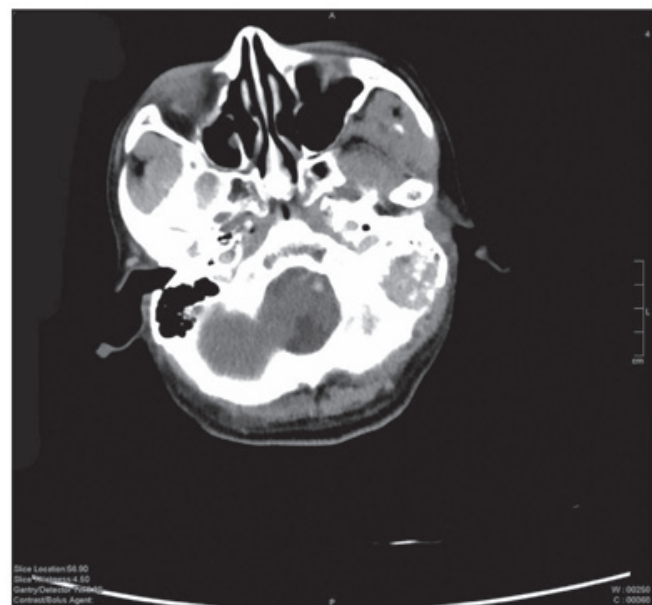

Figure 5. Non-enhanced computed tomography of the temporal bone demonstrated bony destruction in the left temporal bone.

A

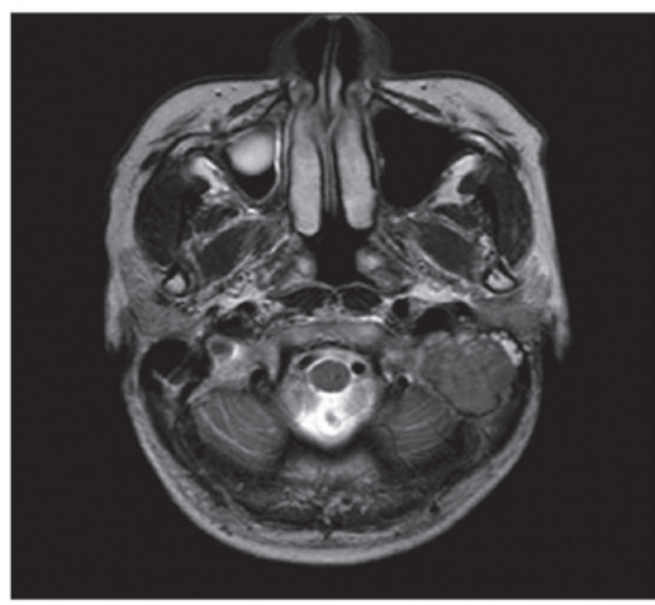

B

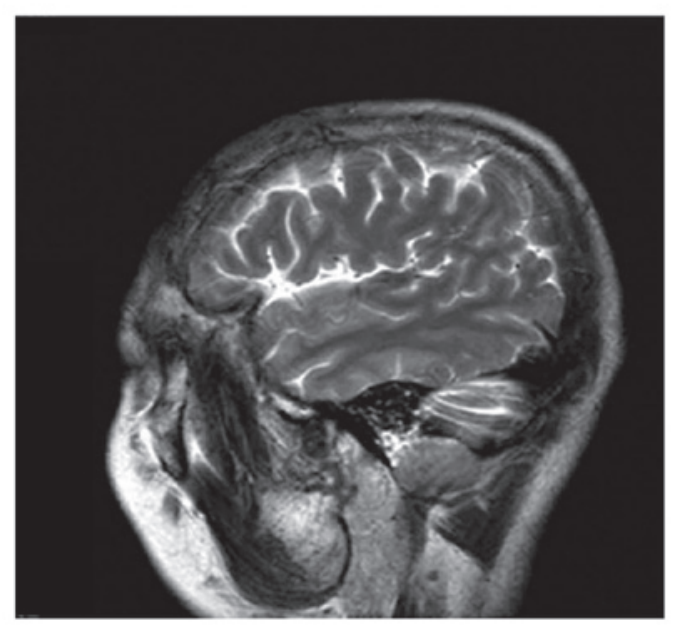

Figure 6. Magnetic resonance imaging identified a diffuse mass in the left temporal bone. The signal corresponding to the mass lesion displayed low intensity on T2-weighted images. (A) Transversal section. (B) Median sagittal section.

surrounding tissues; iii) spreading through the cerebrospinal fluid from an intracranial tumor; iv) leptomeningeal extension; and v) leukemic infiltration. Hematogenous spreading appears to be the most common route of tumor spreading, 
since it was responsible for 11 of the 12 cases reported in a previous study by Belal $\mathrm{Jr}$ (17). In the present case, the symptom of hearing loss may have been caused by direct invasion of the estrous apex through hematogenous spreading from the gastric cancer.

All patients with suspected temporal bone cancer should be subjected to a full neurologic evaluation, including hearing test, electronystagmography and cerebrospinal fluid examination, which may aid in the diagnosis of meningeal carcinoma (16). Radiological imaging, including high-resolution CT and contrast-enhanced MRI are of importance, since the anatomy of the temporal bone hinders physical examination (13). Contrast-enhanced MRI is capable of revealing the extent of the tumor and differentiating the tumor from non-neoplastic soft tissue, while CT scanning enables a reliable assessment of any invasion of the wall of the internal carotid artery canal, jugular bulb and otic capsule (13). The patient of the present case report underwent CT, MRI and bone scan, and was diagnosed with metastasis of gastric cancer to the temporal bone.

The prognosis of temporal bone carcinoma is associated with the stage of the tumor and the effectiveness of the primary treatment (16). The recommend treatment for temporal bone cancer varies widely, and surgery, radiotherapy and chemotherapy should be all considered, depending on the performance status of the patient (6). There is previous evidence of systemic chemotherapy being effective in metastatic disease $(6,16,18)$. Previous studies have reported that chemotherapy combined with 5-FU and cisplatin did not increase the survival rate of patients with temporal bone cancer, but resulted in an improved control of the pain in these patients $(5,6,13)$. Furthermore, previous studies have reported a positive effect of radiotherapy for advanced (T2-T4, according to the Pittsburgh staging system) temporal bone malignancies $(6,13)$.

In conclusion, metastatic tumor of the temporal bone with a history of Her-2 $2^{-}$gastric cancer is rare. Radiological imaging, including MRI, CT, bone scan and positron emission tomography-CT may aid to delineate the extent of metastases.

\section{References}

1. Redon R, Ishikawa S, Fitch KR, Feuk L, Perry GH, Andrews TD, Fiegler H, Shapero MH, Carson AR, Chen W, et al: Global variation in copy number in the human genome. Nature 444: 444-454, 2006.
2. Carcas LP: Gastric cancer review. J Carcinog 13: 14, 2014.

3. Lim ST, Hee SW, Quek R, Lim LC, Yap SP, Loong EL, Sng I, Tan LH, Ang MK, Ngeow J, et al: Comparative analysis of extranodal NK/T-cell lymphoma and peripheral T-cell lymphoma: Significant differences in clinical characteristics and prognosis. Eur J Haematol 80: 55-60, 2008.

4. Lee HJ, Yang HK and Ahn YO: Gastric cancer in Korea. Gastric Cancer 5: 177-182, 2002.

5. Streitmann MJ and Sismanis A: Metastatic carcinoma of the temporal bone. Am J Otol 17: 780-783, 1996.

6. Choi SH, Park IS, Kim YB and Hong SM: Unusual presentation of a metastatic tumor to the temporal bone: Severe otalgia and facial paralysis. Korean J Audiol 18: 34-37, 2014

7. Fukuchi M, Ishiguro T, Ogata K, Kimura A, Kumagai Y, Ishibashi K, Ishida H, Kuwano $\mathrm{H}$ and Mochiki E: Risk factors for recurrence after curative conversion surgery for unresectable gastric cancer. Anticancer Res 35: 6183-6187, 2015.

8. Baiocchi GL, Marrelli D, Verlato G, Morgagni P, Giacopuzzi S, Coniglio A, Marchet A, Rosa F, Capponi MG, Di Leo A, et al: Follow-up after gastrectomy for cancer: An appraisal of the Italian research group for gastric cancer. Ann Surg Oncol 21: 2005-2011, 2014.

9. Satoh H, Okhi T, Momma T, Saito S, Niizeki H, Hirose S and Kuramochi S: Penile skin metastasis of gastric carcinoma associated with prostate carcinoma: A case report. Nihon Hinyokika Gakkai Zasshi 92: 628-631, 2001 (In Japanese).

10. Nakabayashi H, Murata K, Sakaguchi M, Nakajima K and Katsuyama J: Choroid plexus metastasis from gastric cancer - case report. Neurol Med Chir (Tokyo) 34: 183-186, 1994.

11. Shimoyama S, Seto Y, Aoki F, Ogawa T, Toma T, Endo H, Itouji T and Kaminishi M: Gastric cancer with metastasis to the gingiva. J Gastroenterol Hepatol 19: 831-835, 2004.

12. Ohno T, Yokoyama Y, Aihara R, Mochiki E, Asao T and Kuwano H: Sudden bilateral sensorineural hearing loss as the presenting symptom of meningeal carcinomatosis of gastric cancer: Report of a case. Surg Today 40: 561-565, 2010.

13. Kim CH, Shin JE, Roh HG, Lee JS and Yoon SY: Sudden hearing loss due to internal auditory canal metastasis of Her2-positive gastric cancer: A case report. Oncol Lett 8: 394-396, 2014.

14. Joo HY, Chae MH, Lim JH, Yi HG, Lee MH, Kim CS and Park YH: A case of gastric cancer manifesting as a solitary brain metastasis in the cerebellopontine angle that mimicked acoustic neuroma. Chonnam Med J 49: 133-135, 2013.

15. Han JK, Park KH, Lee MS and Lee CK: Gastric carcinoma metastasis to the internal auditory canal. Otol Neurotol 33: e19-e20, 2012.

16. Lionello M, Stritoni P, Facciolo MC, Staffieri A, Martini A, Mazzoni A, Zanoletti E and Marioni G: Temporal bone carcinoma. Current diagnostic, therapeutic, and prognostic concepts. J Surg Oncol 110: 383-392, 2014.

17. Belal A Jr: Metastatic tumours of the temporal bone. A histopathological report. J Laryngol Otol 99: 839-846, 1985.

18. Kunst H, Lavieille JP and Marres H: Squamous cell carcinoma of the temporal bone: Results and management. Otol Neurotol 29: $549-552,2008$. 